\title{
Aplicación del MDN al estudio de la corrosión de los convertidores Peirce - Smith
}

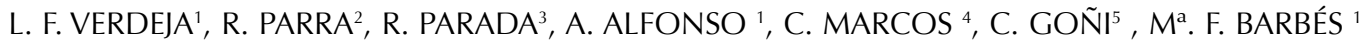 \\ 'Universidad de Oviedo. Escuela de Minas. Siderurgia. Oviedo 33004. España. \\ ${ }^{2}$ Universidad de Concepción. Facultad de Ingeniería. DIMET Concepción. Chile. \\ ${ }^{3}$ Compañía Minera Disputada de Las Condes S.A. Santiago. Chile . \\ ${ }^{4}$ Universidad de Oviedo. Departamento de Geología. Oviedo 33005. España. \\ ${ }^{5}$ Universidad Católica del Norte. Dep. de Ing. Metalúrgica. Antofagasta, Chile.
}

El Modelo de Desgaste Nodal (MDN) constituye una herramienta de trabajo aplicable al estudio de los procesos de corrosión diferencial en los convertidores Peirce - Smith (CPS) con el propósito de alcanzar mejoras significativas en duración y productividad de las diferentes alternativas que se puedan manejar del revestimiento. La aplicación del MDN al CPS precisa del conocimiento de los mecanismos de desgaste existentes y la definición de las ecuaciones de corrosión. Con el MDN se interpretan los resultados de corrosión en la zona de toberas del CPS.

Palabras Clave: Convertidor Peirce-Smith. Desgaste. Modelo de Desgaste Nodal.

\section{Nodal Wear Model in the corrosion Peirce - Smith copper convertor}

The Nodal Wear Model (NWM) constitutes a tool of applicable work study the processes of differential corrosion in the Peirce - Smith convertor (PSC) with the purpose of reaching significant improvements in duration and productivity of the several alternatives that one could utilize of the coating.

For the application of the NWM to CPS is necessary of the knowledge the mechanisms of existent waste and the definition of the corrosion equations. With the NWM the outputs of corrosion in the tuyere zone of CPS are interpreted.

Key words: Peirce - Smith convertor. Corrosion. Nodal Wear Model (NWM).

\section{INTRODUCCIÓN}

Uno de los procesos básicos de la pirometalurgia del cobre consiste en el tratamiento de la mata de cobre o del metal blanco en cobre blister en los convertidores Peirce-Smith (CPS) ,Figura 1. El aumento de la productividad de las instalaciones pirometalúrgicas de obtención de cobre gravita en la transformación discontinua de las operaciones en labores semicontinuas o continuas. Bajo estas circunstancias, los materiales utilizados para los revestimientos han de ser capaces de garantizar un mínimo de horas de trabajo bajo condiciones de máximo esfuerzo térmico.

Se desarrolla en el trabajo las primeras propuestas de las ecuaciones de corrosión que puedan incorporarse al estudio del desgaste del CPS siguiendo el sistema de cálculo que ofrece el MDN (1), (2) y (3).

\section{EL MODELO DE DESGASTE NODAL: FUNDAMEN- TOS TEÓRICOS}

La determinación en estado estacionario del perfil térmico del revestimiento refractario de un convertidor permite conocer la temperatura en la interfase fundido/refractario (nodal) partiendo de unas condiciones límites conocidas, Figura 2. La temperatura o flujo de calor a lo largo de la coraza externa del CPS es una de las condiciones límites del problema y que además puede ser medida de forma experimental. Otra condición de contorno necesaria para la solución del problema térmico hace referencia al coeficiente de transporte convectivo, $h$, entre el fundido y el refractario. Este coeficiente puede calcularse de forma empírica, por ejemplo, conociendo los valores de que alcanza la temperatura en diferentes alturas del revestimiento. Con estos datos, en estado estacionario se determina, conociendo la conductividad térmica de los materiales, el flujo de calor en el refractario y por lo tanto el que se transmite desde el fundido hacia el refractario.

Sin embargo, el fenómeno de corrosión modifica la geometría del sistema, por lo tanto, la solución del problema térmico transcurrido un $\Delta t$ (o un número de ciclos $N$ ) no es válida ya que una nueva distribución de temperatura se ha ido consolidando a lo largo de la solera del convertidor. El cálculo de la nueva geometría se obtiene aplicando a cada uno de los nodos de la intercara del refractario con el fundido la correspondiente ecuación de corrosión. Esta ecuación, característica según el mecanismo controlante del proceso de desgaste, es determinada de forma fenomenológica o semiempírica. Por otra parte, la ecuación de corrosión viene a ser en definitiva una función de la temperatura nodal y de la diferencia de temperatura entre este y sus nodos adyacentes o entre el nodo de la intercara considerada y la temperatura del fundido, Figura 3 . Es decir, la velocidad de corrosión en el nodo $i$ de una intercara, expresada en unidades de longitud por unidad de tiempo:

$$
v(\text { corrosión })_{i}=f\left(T_{i} ; \Delta T_{i}\right)
$$

\section{ECUACIONES DE CORROSIÓN}

Una de las zonas de máximo desgaste en los CPS es la situada alrededor de las toberas, Figuras 1 y 2 . La duración del material refractario en esa zona repercute en la productividad de la conversión. Se ha considerado, a tenor de los resultados que hasta el momento se tienen de los estudios "post mortem" del material de toberas (4),(5) y (6), que los mecanismos involucrados en el desgaste de los refractarios de magnesia - cromo pueden ser los siguientes:

1. Penetración del fundido a través de la porosidad abierta del refractario (Mecanismo Capilar). 
2. Ataque químico del constituyente matriz del refractario por el fundido (Mecanismo Químico). No obstante, la etapa lenta del proceso es la difusión de los productos a través de la costra, Figura 3.

3. Fatiga térmica a lo largo de la intercara costra - refractario producto de la penetración del fundido y de los ciclos de carga - colada del convertidor durante el proceso de obtención del cobre blister, Figura 3, (Mecanismo de Fatiga Térmica).

\subsection{Mecanismo Capilar}

El desarrollo matemático del Mecanismo Capilar en la corrosión de materiales refractarios ha sido publicado en la referencia (2). Es preciso destacar en este caso que es más importante conocer la profundidad de la penetración del fundido, $e_{c}$, que la velocidad bajo la cual se produce. Por otra parte, el tiempo que precisa la infiltración para desarrollarse es muy pequeño si se compara con el que necesita el proceso de corrosión química o de fatiga térmica. El conocimiento de las características físico - químicas del fundido (posible existencia de afinidades eutécticas) será un instrumento básico de apoyo en el cálculo teórico de los espesores de infiltración, Figura 3.

\subsection{Mecanismo Químico}

La ecuación que representa la velocidad de corrosión del refractario en metros por segundo, actuando como etapa lenta del proceso la difusión de las especies atacadas a través de la costra, Figura 3,es semejante a la utilizada para la simulación del desgaste en el crisol del alto horno (1) y (3):

$$
v(\text { corrosion })_{i, D}=2,5010^{-3} L^{-1 / 2} \mu_{i}^{-1 / 6} \rho_{i}^{1 / 6} D_{i}^{11 / 12} \Delta(\% C)_{i}\left(\frac{\rho_{i}}{\rho_{g}}\right)\left(\frac{100}{\% c m}\right)
$$

si bien en este caso, $L$, es la profundidad alcanzada por el fundido en el refractario se identifica con $e_{c^{\prime}}$ Figura 3. Igualmente, $\mu_{\mathrm{i}}$, representa la viscosidad nodal del fundido, $\rho_{i^{\prime}}$ la densidad nodal del fundido, $D_{i^{\prime}}$ el coeficiente de difusión nodal del compuesto resultante del ataque químico a través de la costra, $(\Delta \% \mathrm{C})$, el gradiente de concentración del compuesto resultante del ataque químico a lo largo de la costra en el nodo $i, \rho_{g}$, la densidad global de refractario y $\% \mathrm{~cm}$, el porcentaje del constituyente matriz en la estructura del refractario utilizado para el revestimiento.

\subsection{Mecanismo de Fatiga Térmica}

La derivación de la ecuación que representa la magnitud del avance de una grieta en la intercara costra - refractario, Figura 3, a partir de un defecto preexistente en el material (poros), $\Delta a$, tiene su fundamento en un balance energético: la energía necesaria para crear una nueva intercara sólido - gas ( que depende del valor de la energía superficial, $\gamma_{\mathrm{sg}}$, y de la microestructura, $A / V$, área - volumen de la matriz y el disperso del refractario) es igual al trabajo de las fuerzas desarrolladas en la intercara costra - refractario que aparecen como consecuencia de los ciclos térmicos del sistema, $N$, y de las distintas propiedades termo - mecánicas de los materiales que conforman la costra y el refractario ( ver características de los símbolos utilizados por la ecuación [3] en la Figura 3):

$$
\Delta a=C\left(\alpha_{c}-\alpha_{r}\right) \Delta T_{i} E_{c} e_{c} \frac{1}{\left[\gamma_{s / g}\left[\frac{A}{V}\right]\right]_{r e f}}\left[\frac{1}{N}\right]
$$

en donde $C$, es una constante de proporcionalidad adimensional correctora de todas aquellas simplificaciones introducidas en la derivación de la expresión [3].

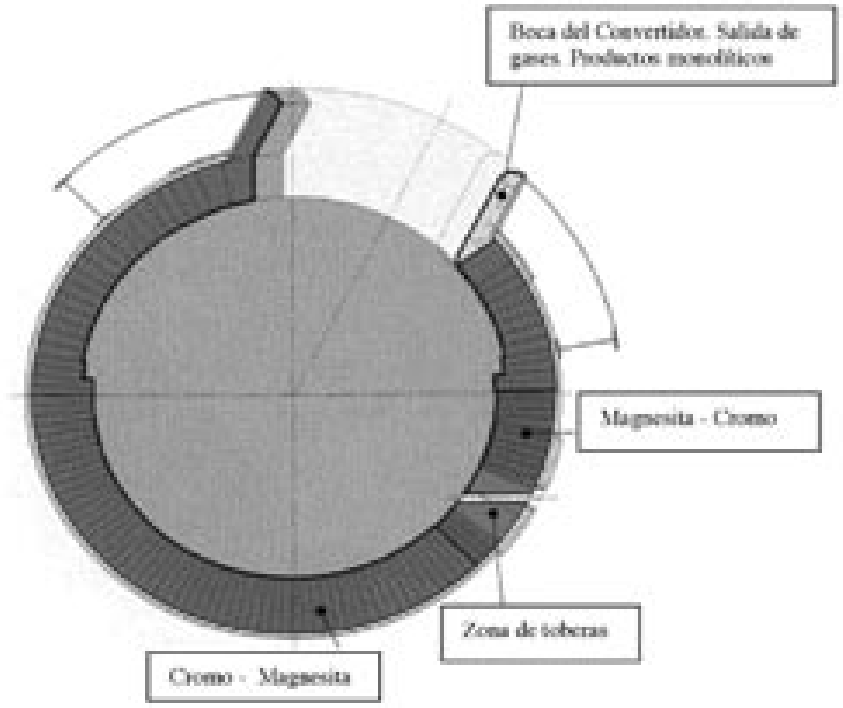

Figura 1. Sección transversal de un CPS.

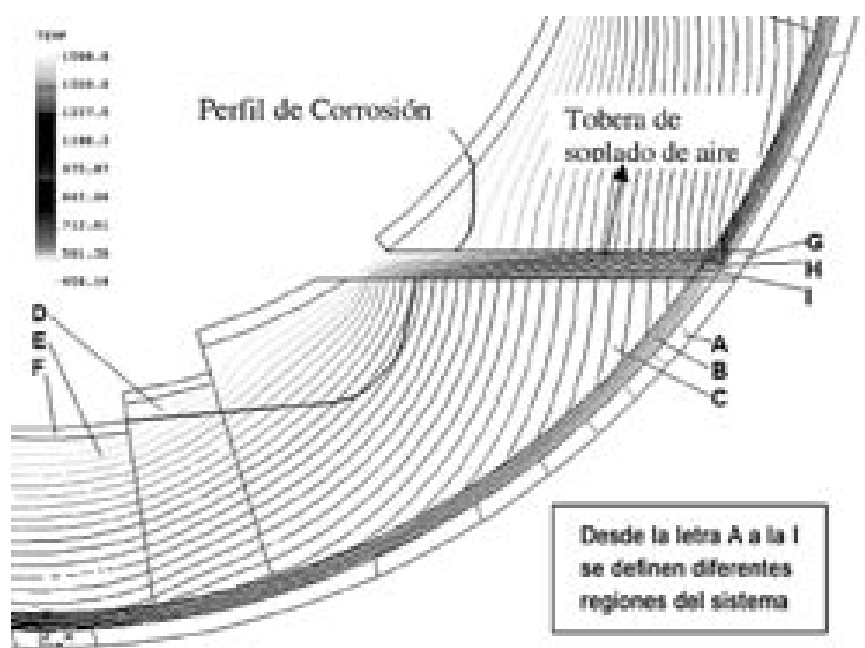

Figura 2. Distribución de temperaturas alrededor de la tobera (del documento presentado por los autores del trabajo al Cobre-203)

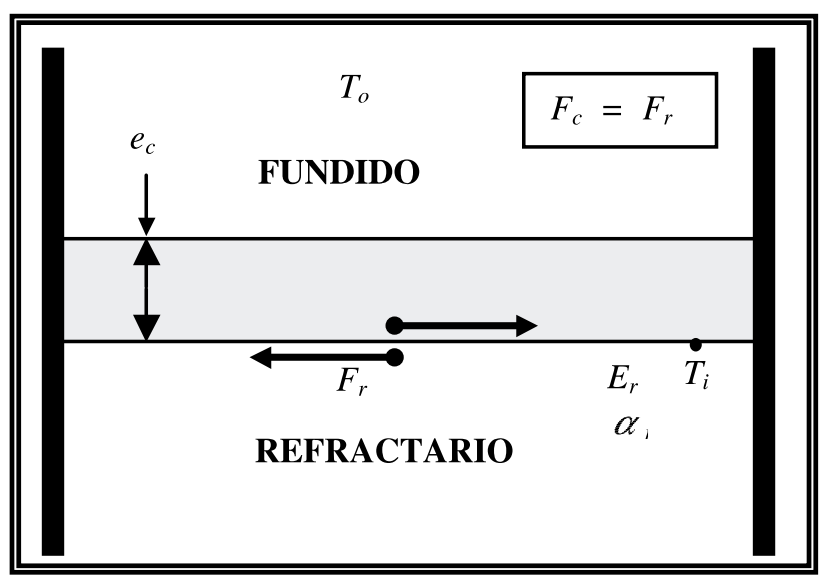

$\alpha_{\mathrm{r}}$ (Coeficiente de dilatación del refractario) ; $\alpha_{c}$ (Coeficiente de dilatación de la costra) $E_{\mathrm{r}}$ (Módulo elástico del refractario); $E_{\mathrm{r}}$ (Módulo elástico de la costra)

$\mathrm{T}_{0}$ (Temperatura del fundido); $\mathrm{T}_{\mathrm{i}}$ (Temperatura de la intercara) $e_{c}$ (Espesor de la costra)

Figura 3. Mecanismo de desgaste por Fatiga Térmica de materiales refractarios. 


\section{RESULTADOS EXPERIMENTALES}

Una de las propuestas para evaluar la corrosión en la zona de toberas del CPS pasa por representar la disminución del espesor de las paredes refractarias en función del número de cargas de mata o de coladas de cobre blister ejecutadas, Figura 4. Puede interpretarse la curva de desgaste, a tenor de lo desarrollado por las consideraciones teóricas del MDN, apartados 2 y 3, de la siguiente manera:

1. Durante las primeras 62 cargas, el material refractario aparentemente permanece inalterado. No obstante durante el citado periodo tiene lugar la infiltración capilar del fundido y el ataque químico a la matriz del refractario que refuerza las posiciones alcanzadas por fundido que se ha infiltrado a través de la porosidad abierta. Se forma una costra con material fundido infiltrado y los productos de corrosión de la matriz refractaria, sobre el material sin atacar, Figura 3.

2. Entre las coladas 62 y 80, el mecanismo responsable de la mayoría del desgaste es la Fatiga Térmica (ecuación [3]). Se desprenden súbitamente $40 \mathrm{~mm}$. de pared, magnitud que se identificaría con el espesor alcanzado por la costra, , generada por el Mecanismo Capilar y Químico durante las primeras 62 cargas.

3. A partir de la carga 80 , sucede un nuevo proceso de infiltración capilar y el correspondiente ataque químico de la matriz refractaria controlado por la difusión de los productos de la corrosión a través e la costra, ecuación [2].

4. Desde la carga 95, además del mecanismo de desgaste químico, se produce una nueva rotura por Fatiga Térmica del revestimiento equivalente a un espesor de costra comprendido entre los 25 y $30 \mathrm{~mm}$.

5. Entre la carga 140 y la 295, el mecanismo predominante es la difusión de los productos del ataque químico a través de la costra. En este caso el espesor de la misma no es tan importante como en los dos casos anteriores (carga 65 y carga 95): puede ser tan solo de algunos milímetros ( entre 3 y $10 \mathrm{~mm}$.).

6. A partir de la colada 295, tiene lugar un nuevo desgaste por $F a$ tiga Térmica (entre 3 y $10 \mathrm{~mm}$.) aunque en esta ocasión bastante menos importante que en las dos situaciones anteriores.

7. Finalmente, hasta el final de la vida operativa del refractario de toberas, el Mecanismo Químico, es el controlante del proceso de desgaste de los materiales.

Puede calcularse, a partir de los fundamentos teóricos desarrollados en el apartado 3.3, que la tensión localizada a lo largo de la costra es superior a la existente en el refractario. No obstante, si el espesor de la costra es muy importante, las tensiones que aparecen en el refractario pueden superar el valor crítico de fractura. Por esta circunstancia, cuando el espesor de la costra es muy grande, la fatiga térmica provoca la fractura del material (desprendimiento) con valores que no se corresponde con el espesor de la capa infiltrada: normalmente bastante superiores a $e_{c}$, Figura 3.

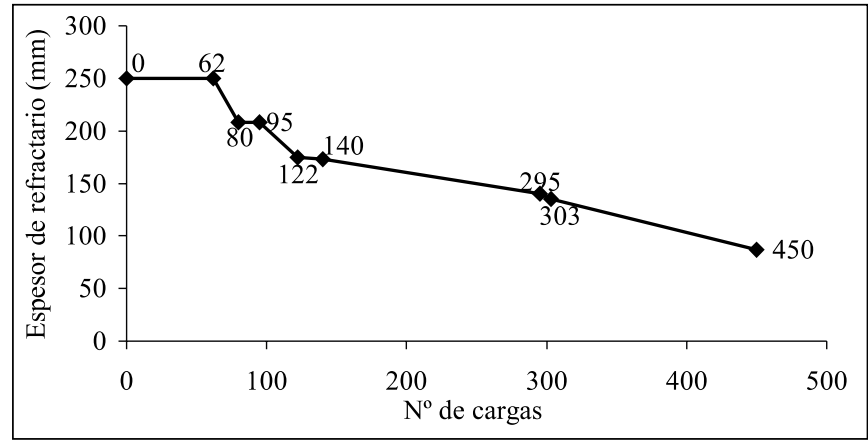

Figura 4. Variación del espesor refractario en toberas en función del número de operaciones de carga.

\section{CONCLUSIONES}

El MDN constituye una alternativa válida para el estudio de las corrosiones diferenciales que se dibujan en los revestimientos de $\mathrm{MgO}$ - $\mathrm{Cr}_{2} \mathrm{O}_{3} \cdot \mathrm{FeO}$ de los CPS, Figura 2. Se proponen las ecuaciones de corrosión que han de manejarse para simular el desgaste del refractario: Mecanismo Capilar, Químico y Fatiga Térmica.

Igualmente, el MND resulta un instrumento de gran utilidad para estudiar - simular todas aquellas propuestas alternativas para los revestimientos de los CPS que intenten mejorar la productividad de las instalaciones.

\section{AGRADECIMIENTOS}

Para el Ministerio de Ciencia y Tecnología ( MAT2000-1715) y a la Disputada de la Condes - Chile por las ayudas recibidas para el desarrollo de las investigaciones.

\section{BIBLIOGRAFÍA}

1. L. F. Verdeja, A. Alfonso, R. González, "Material wear in blast furnace crucibles", Am. Ceram. Soc. Bull., 77 [5] 91-96 (1998).

2. L. F. Verdeja, R. Parra, J. P. Sancho, J. Bullón, “Corrosion mechanism and wear prediction of the sole of an electric arc furnace",ISIJ, 43[2]192200(2003).

3. L. F. Verdeja, R. González, A. Alfonso, Ma . F. Barbés, “Nodal wear model: corrosion in carbon blast furnace hearths", Rev. Metal, Madrid, 39[3]183192(2003).

4. J. L. Liow, P. Tsirikis, N. B. Gray, "Study of refractory wear in the tuyere region of a Peirce - Smith nickel convertor", Canad. Metall. Quart., 37 [2] 99-117 (1998).

5. C. Marcos, M. A.Llorca, P. Camblor, L. F. Verdeja, “Caracterización de refractarios monolíticos", Bol. Soc. Esp. Ceram. V., 41 [2] 241-251 (2002).

6. M. A. Fernández, “Consideraciones sobre el mecanismo de desgaste refractario en los convertidores Peirce - Smith". Proyecto Fin de Carrera. Ingeniería Química. Universidad de Oviedo, ( 2002), p. 71.

Recibido: 1.2 .03

Aceptado: 30.11 .03 